\title{
Can we improve the outcome of colorectal cancer by early diagnosis?
}

\section{E D A Westcott, A C J Windsor}

Screening, not early diagnosis, may be the best approach

$\square$ olorectal cancer is the second commonest cause of cancer in the UK with over 30000 new cases each year. The overall five year survival rate of $40 \%$, ${ }^{1}$ together with well published regional variations in outcome, increased public awareness and expectation have prompted a careful look at the management of this disease. Late presentation remains one of the key areas of concern, and there are a number of proposed approaches designed to increase the proportion of patients for whom cure is a realistic goal. The first is to offer screening to the population as a whole, the second to offer screening to individuals in high risk groups, and the third rapid diagnosis of symptomatic patients.

Colorectal cancer readily fulfils the criteria for mass screening: a high incidence, outcome affected by stage of disease and modified by early intervention, and adenomatous polyps as an identifiable precancerous lesion. However, a truly appropriate and valid screening test remains elusive and the ability of an overstretched health care system to provide such a "gold standard" investigation remains in doubt.

In 1996 the Nottingham group screened 152850 participants with faecal occult blood tests and increased the proportion of patients with Dukes A carcinoma in the screened population from $11 \%$ to $20 \%$ and decreased the percentage of patients with stage $\mathrm{C}$ or D from $52 \%$ to $46 \%$ compared with controls. The resulting reduction in disease specific mortality from $49 \%$ to $40 \%$ was, however, offset by a disappointing test sensitivity and patient compliance of only $50 \%{ }^{2}$ Mandel et al followed up a cohort of screened individuals for 18 years with either annual or biennial faecal occult blood tests and demonstrated a significant reduction in cumulative incidence of colorectal cancer and an overall reduction in incidence of $20 \%$ compared with the control group. ${ }^{3}$

Full colonoscopy is perhaps the most sensitive investigation for identifying polyps in the large bowel. However, it has a small but significant complication rate, ${ }^{4}$ and a caecal intubation rate of only $57.1 \%$ if ileocaecal valve visualisation or terminal ileum intubation are regarded as the only reliable methods of identifying a complete procedure..$^{5}$ Flexible sigmoidoscopy, while attractive because of its simplicity, will undoubtedly fail to detect at least $25 \%$ of significant adenomas. In addition, Lieberman and Weiss compared faecal occult blood tests and flexible sigmoidoscopy with colonoscopy in asymptomatic individuals between the ages of 50 and 75 years. They demonstrated that $24 \%$ of advanced colonic neoplasia would be missed had they relied on faecal occult blood and flexible sigmoidoscopy alone. However, its ability to predict more proximal lesions and the subsequent development of malignancy is the subject of a recently completed randomised controlled trial in an asymptomatic population between the ages of 55 and 64. The first analysis of colorectal cancer incidence and mortality data are due in 2004, ${ }^{7}$ and the results are awaited with interest.

With regard to high risk populations such as those with familial adenomatous polyposis (FAP) or ulcerative colitis, and patients with a family history of colorectal cancer, screening is already undertaken, to varying degrees. The protocol used at St Mark's Hospital for patients with a history of FAP is first colonoscopy at the age of 15 with dye spray. If the patient is affected, colectomy and ileorectal anastomosis are undertaken in the late teens for low density polyposis $(<1000$ polyps overall $)$ and restorative proctocolectomy offered to those with high density polyps (>1000 polyps). Surveillance for patients with extensive ulcerative colitis remains controversial. While annual or biannual surveillance with random biopsies is commonly practised, there is evidence that many cancers are diagnosed outside the existing surveillance programmes. ${ }^{8}$ Family history of colorectal cancer is a source of concern to relatives. A single first degree relative certainly increases an individual's risk of developing colorectal cancer, but once again the logistics of screening all relatives within an already stressed health service has meant refining the indications. Currently the recommendation is to screen relatives with a family pedigree that conveys a risk of greater than one in 10. Thus individuals with two or more first degree relatives who have had colorectal cancer and/or those with one first degree relative who presented before the age of 50 should receive colonoscopic screening starting five years before the age at which their relative first presented. Depending upon the findings a surveillance interval of between three to five years is then recommended.

The third strategy to improve outcome is rapid or early diagnosis of symptomatic patients. The validity and effectiveness of this approach must first question whether a delay in diagnosis or treatment is likely to have a detrimental effect on outcome and at what stage in the natural history of the disease a tumour becomes incurable. A small number of studies do show a correlation between short duration of symptoms and favourable outcome, however other studies have shown an inverse correlation.' Biological predeterminism suggests that clinical outcome is predicted by tumour biology, thus chronic symptoms are attributable to slow growing favourable tumours, while acute symptoms predict an aggressive, less favourable pathology. Furthermore, the biology of the tumour may predict when it metastasises, thus an aggressive tumour biology may allow for early metastases before the development of symptoms, therefore preventing effective cure even in the face of a rapid diagnostic service. While this may be true, striving for early treatment and diagnosis may serve to improve quality of life by decreasing anxiety of those with both benign and malignant disease even if it does not improve outcome.

Current thinking suggests that symptom complexes or combinations of symptoms yield a higher sensitivity and specificity of diagnosis. The Portsmouth Audit identified a change in bowel habit and rectal bleeding in patients over 55 years, in the absence of abdominal pain and perianal symptoms and signs, as the combination most likely to yield the diagnosis of cancer with a risk of $33.7 \%$. With the addition of abdominal pain to the symptom complex the risk fell to $20.5 \%$ and, with a change in bowel habit, rectal bleeding and perianal symptoms or signs, a diagnosis of cancer was present in only $11.9 \%$ of patients. For patients under the age of 55 the chance of cancer was greatly reduced with even the most worrying combination of symptoms; change in bowel habit and rectal bleeding giving only a $4.9 \%$ chance of having a malignancy. Such combinations of symptoms form the basis of published guidelines for referral from primary care.

What of the future? Given the resources and logistics a valid and compliant screening programme would seem the ideal approach to improving outcome for colorectal cancer. Rapid advancements in helical computed tomography and magnetic resonance imaging, and the advent of protocols that negate the 
use of bowel preparation, may allow rapid and non-invasive screening methods for the future. While we await these developments we must continue to strive with the means currently at our disposal to improve the lot of patients with colorectal malignancy.

Postgrad Med J 2002;78:255-256

\section{Authors' affiliations}

E D A Westcott, A C J Windsor, St Mark's Hospital, Watford Road, Harrow, Middlesex HAI 3UJ, UK

Correspondence to: Mr Windsor

\section{REFERENCES}

1 Rhodes JM. Colorectal cancer screening in the UK: joint position statement by the British Society of Gastroenterology, The Royal College of Physicians, and The Association of Coloproctology of Great Britain and Ireland. Gut 2000;46:746-8.

2 Hardcastle JD, Chamberlain JO, Robinson $\mathrm{MH}$, et al. Randomised controlled trial of faecal-occult-blood screening for colorectal cancer. Lancet 1996:348:1472-7.

3 Mandel JS, Church TR, Bond JH, et al. The effect of fecal occult-blood screening on the incidence of colorectal cancer. N Engl J Med 2000;343: 1603-7.

4 Tran DQ, Rosen L, Kim R, et al. Actual colonoscopy: what are the risks of perforation? Am Surg 2001;67:845-7.

5 Bowles CJA, Leicester R, Swarbrick E, et al. Intercollegiate-BSG National colonoscopy (INBC) audit: methods used to identify the caecum and caecal intubation rate. Gut 2001;48(suppl 1):i10.

6 Lieberman DA, Weiss DG. One-time screening for colorectal cancer with combined fecal occult-blood testing and examination of the distal colon. N Engl J Med 2001;345:555-60.

7 Atkin WS, Edwards R, Wardle J, et al. Design of a multicentre randomised trial to evaluate flexible sigmoidoscopy in colorectal cancer screening. J Med Screen 2001;8:137-44.

8 Mayer $\mathbf{R}$, Wong WD, Rothenberger DA, et al. Colorectal cancer in inflammatory bowel disease: a continuing problem. Dis Colon Rectum 1999:42:343-7.

9 Porta M, Gallen M, Malats N, et al. Influence of "diagnostic delay" upon cancer survival: an analysis of five tumour sites. J Epidemiol Community Health 1991;45:225-30.

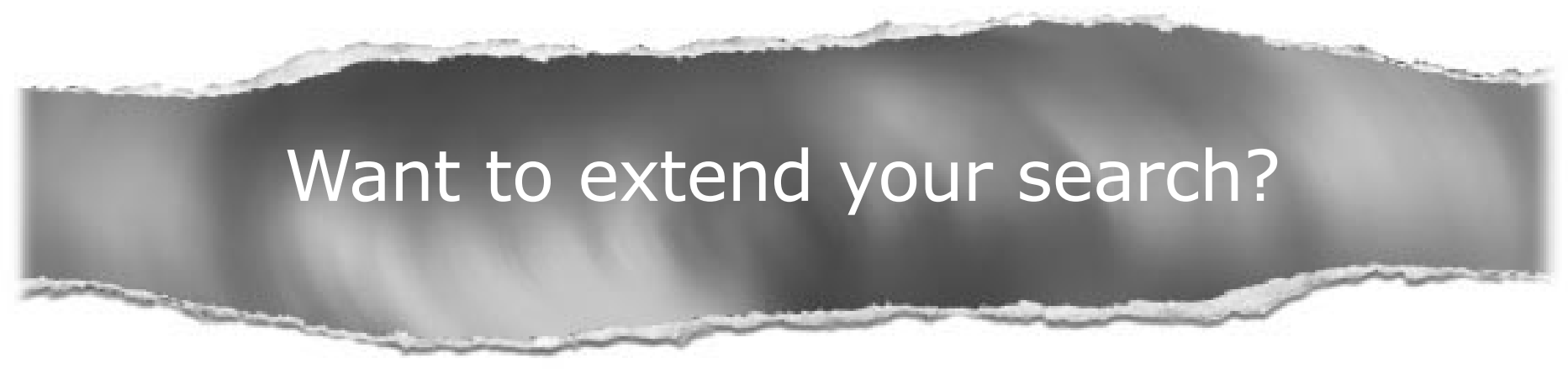

Cross journal searching

If you can't find what you are looking for in the Postgraduate Medical Journal you can extend your search across many of the more than 200 journals available for selection. You can restrict your search to specific subject areas (eg, clinical medicine, basic research), or select specific journals, or search all available titles.

\section{www.postgradmedj.com}

